\title{
Safety and efficacy of digoxin: systematic review and meta-analysis of observational and controlled trial data
}

In this Research paper by Ziff and colleagues (BMJ 2015;351:h4451, doi:10.1136/bmj.h4451) an error was introduced into figure 1 (Selection of studies flowchart (after duplicates removed) on safety and efficacy of digoxin). In the box that lists the number of "full text articles assessed for eligibility," this should have read n=82 [not "n=1916" as was published].

Cite this as: BMJ 2015;351:h4937

๑ BMJ Publishing Group Ltd 2015 